\title{
Eosinophilic Granulomatosis Polyangiitis (EGPA) Masquerading as a Mycotic Aneurysm of the Abdominal Aorta: Case Report and Review of Literature
}

\author{
Pooja Kumari $\mathbb{D}^{1},{ }^{1}$ Debendra Pattanaik $\left(\mathbb{D},{ }^{1}\right.$ and Claire Williamson $\mathbb{D}^{2}$ \\ ${ }^{1}$ Division of Rheumatology, Department of Medicine, University of Tennessee Health Science Center, Memphis, TN 38163, USA \\ ${ }^{2}$ Department of Pathology and Laboratory Medicine, University of Tennessee Health Science Center, Memphis, TN, USA \\ Correspondence should be addressed to Debendra Pattanaik; dpattana@uthsc.edu
}

Received 8 August 2021; Accepted 1 September 2021; Published 13 September 2021

Academic Editor: Gregory J. Tsay

Copyright (C) 2021 Pooja Kumari et al. This is an open access article distributed under the Creative Commons Attribution License, which permits unrestricted use, distribution, and reproduction in any medium, provided the original work is properly cited.

\begin{abstract}
Introduction. Aortic involvement leading to aortitis in eosinophilic granulomatosis polyangiitis (EGPA) is infrequent, and only 2 cases have been reported so far in the literature. Even more so, aortic aneurysm, secondary to EGPA, has never been reported and remains a diagnostic and therapeutic challenge. Case Presentation. We present a 63-year-old Caucasian male patient with a prior diagnosis of EGPA presenting with abdominal pain, nausea, and loose stools to the emergency department. Physical examination showed periumbilical tenderness. He had no peripheral eosinophilia but had high C-reactive protein and procalcitonin levels. CT abdomen revealed a mycotic aneurysm involving the infrarenal abdominal aorta. The patient declined surgical repair initially and was treated with IV antibiotics only. Unfortunately, 24 hours later, the aneurysm ruptured, leading to emergent axillofemoral bypass surgery. Surgical biopsy showed aortitis, periaortitis, and active necrotizing vasculitis. Conclusion. Abdominal aneurysms should be considered a complication of EGPA, and earlier immunosuppressive therapy should be considered to prevent further complications.
\end{abstract}

\section{Introduction}

Previously called Churg Strauss syndrome (CSS), eosinophilic granulomatosis with polyangiitis (EGPA) is a subtype of antineutrophil cytoplasmic antibody- (ANCA-) associated vasculitis (AAV) [1]. It is characterized by eosinophilic necrotizing inflammation involving medium- and small-size vessels [1]. Involvement of large vessels is sporadic, with only 2 cases being reported presenting as aortitis in the literature. There is no reported case of aortic aneurysm in EGPA. We report here the first case of abdominal aortic aneurysm secondary to EGPA masquerading as a mycotic aneurysm, leading to delay in diagnosis and appropriate treatment.

\section{Case Presentation}

A 63-year-old Caucasian male subject presented to GI clinic in May 2009 with complaints of bloating, constipation, and
30 pounds weight loss for the past 6 months. He denied any abdominal pain, nausea, vomiting, dysphagia, diarrhea, hematochezia, or rash. Past medical history includes allergic rhinitis, chronic sinusitis, hearing loss, psychotic, and paranoid personality disorder. He had an appendectomy before. He denied smoking, alcohol, or any illicit drug use. Physical examination revealed nasal polyps, but the rest of the examination was unremarkable. Complete blood count showed peripheral blood eosinophilia of $53.1 \%(0.1-8)$, elevated erythrocyte sedimentation rate (ESR) of $31 \mathrm{~mm} /$ hour $(0-20)$, and C-reactive protein (CRP) of $22.7 \mathrm{mg} / \mathrm{L}(0.0-4.0)$. Liver function tests (LFTs) showed elevated alkaline phosphatase of $285 \mathrm{U} / \mathrm{L}$ (40-140) and elevated gamma-glutamyl transferase (GGT) of $135 \mathrm{U} / \mathrm{L}$ (10-75). Antinuclear antibody (ANA), complements, cytoplasmic antineutrophilic antibodies (c-ANCA), proteinase3 (PR3) antibody, and myeloperoxidase (MPO) antibody were all normal. However, perinuclear antineutrophil cytoplasmic antibody ( $\mathrm{p}$-ANCA) 
was positive in low titer: $1: 80$. Stools for ova and parasites were negative but had a positive toxocara antibody. He was treated with ivermectin without any improvement in symptoms. Subsequently, a bone marrow biopsy was done and was negative for any myeloproliferative disorders. Biphasic liver CT showed diffuse portal fibrosis concerning biliary cirrhosis. A duodenal biopsy revealed areas with profound eosinophilic infiltrate (>100 eosinophils/HPF) suggestive of eosinophilic enteritis. A liver biopsy showed multiple hepatic granulomas with central necrosis and extensive eosinophilia. Based on the constellation of symptoms and biopsy findings, he was diagnosed with EGPA. Treatment with high-dose steroids was recommended, but the patient declined immunosuppressive medications due to concern for side effects. He continued to follow with allergy/ immunology and rheumatology service and had multiple hospitalizations for asthma flare. He was receiving treatment with on and off corticosteroids for asthma flare and inhalers for asthma. In January 2020, he presented to the emergency department (ED) with a 2-week history of sudden onset sharp and right-sided lumbar back pain, which started after moving a large spare tire. He also reported pain in the umbilical region aggravated by meals and associated with nausea and loose stools. Vital signs were as follows: Blood pressure $129 / 77 \mathrm{~mm}$ of hg, pulse was 79/minute, RR was 12/ minute, and temperature was $36.6^{\circ} \mathrm{C}$. Physical examination was significant only for periumbilical tenderness. Laboratory tests showed white blood cell count of 14,240/ul (4000-10,000) and differentials of eosinophils $2 \%(0.1-8)$, polymorphonuclear cells $82 \%$ (38-72), and lymphocytes $8.3 \%$ (15-45). Hemoglobin and platelet counts were normal. CRP was $104 \mathrm{mg} / \mathrm{L}(0.0-5.0)$ and procalcitonin was $7.4 \mathrm{ng} /$ $\mathrm{mL}(0.0-0.25)$. Renal function and urinalysis were normal. The liver function test showed normal transaminases, but alkaline phosphatase was elevated at $217 \mathrm{U} / \mathrm{L}$ (40-150). ANCA panel and ANA were negative. IgG4 was minimally elevated at $125 \mathrm{mg} / \mathrm{dl}$ (2-96). Blood cultures were negative. CT abdomen and pelvis with and without contrast showed asymmetric fusiform sac measuring $3.6 \times 3.2 \mathrm{~cm}$ along the right lateral and anterior margin of the abdominal aorta at the level of L4. There was aortic wall calcification along the right lateral margin and hazy wall thickening of the aneurysm sac with surrounding stranding and haziness. These are suggestive of mycotic aneurysms (Figures 1 (a) and 1(b)). He was admitted to vascular surgery service. Infectious disease and rheumatology services were consulted. He was started on broad spectrum IV antibiotics, but he refused any surgical intervention for the aneurysm. The following day, the patient had worsening abdominal pain and a repeat CT abdomen with contrast showed localized areas of contrast bulging in the right side of the infrarenal abdominal aorta, projecting into the periaortic soft tissue thickening. These contrast projections extended beyond the calcified wall of the aorta and were contained within the soft tissue thickening; findings were consistent with confined aortic rupture associated with periaortic inflammatory thickening. He was taken to the operating room for an emergent exploratory laparotomy. The infrarenal aorta was resected and a right axillobifemoral bypass was performed with an $8 \mathrm{~mm}$ polytetrafluoroethylene (PTFE) graft. Aortic wall tissue was sent for histopathology analysis. Aortic wall pathology showed markedly inflamed, hemorrhagic, and partially necrotic periaortic and scant aortic tissue infiltration of plasma cells, lymphocytes, and neutrophils and histiocytes with occasional eosinophils within the vessel wall. It also showed eosinophilic vasculitis of mostly small and rarely medium vessels with rare fibrinoid necrosis (Figures 2(a) and 2(b)). No granuloma was identified. Gram stain and Periodic Acid Schiff Stain (PAS) stain for fungus were negative. The final pathologic diagnosis was aortitis, periaortitis, and necrotizing vasculitis suggestive of EGPA. After multiple other postsurgical complications, he had a prolonged and difficult recovery in the surgical intensive care unit. After a month-long recovery period, he was offered further immunosuppressive therapy, which he continues to decline.

\section{Discussion}

We presented a case of long-standing EGPA, presenting to the emergency department with abdominal pain and CT abdomen findings of a mycotic aneurysm involving infrarenal abdominal aorta. Unfortunately, the aneurysm ruptured, and emergent surgery was performed. The resected aortic tissue showed aortitis, periaortitis, and necrotizing vasculitis. Postoperatively patient had a slow prolonged recovery, and he continued to decline further immunosuppressive treatment.

Granulomatosis polyangiitis (GPA), microscopic polyangiitis (MPA), and EGPA are classified as AAV involving small- and medium-size vessels [1]. Several case series have reported the involvement of large vessels in patients with GPA and MPA [2-4]. However, large vessel involvement including the aorta resulting in aortitis has been reported only in 2 cases of EGPA who were newly diagnosed but none had aortic aneurysm $[5,6]$. Aortic involvement in our case happened almost ten years after the initial diagnosis and resulted in aneurysm formation, unlike in previously 2 reported cases. The pathogenesis of ANCA-associated large vessel disease is unknown. It is believed that ANCAs contribute to the pathogenesis of small and medium vessel vasculitis [7]. ANCAs may be involved in the pathogenesis of large vessel vasculitis as well [3]. Our patient only had low titer $\mathrm{p}$-ANCA positivity on initial presentation ten years ago but was negative afterward including the MPO antibody. One study reported a role of vasovasoritis, e.g., inflammation of small vessels feeding the large arteries, and leading to the development of the large vessel vasculitis [2]. Another case reported only intimal involvement without any involvement of media and adventitia [4]. However, histopathologic analysis of 5 cases revealed transmural aortitis [3]. Based on this evidence, it is possible that intimal injury occurs as the initial component of ANCA-associated large vessel disease and slowly involves the entire vessel wall [3].

Infected aneurysms, otherwise known as "mycotic" aneurysms, and inflammatory aneurysms account for a minority of cases of abdominal aortic aneurysms [8]. Inflammatory aortic aneurysms are usually seen with giant cell 


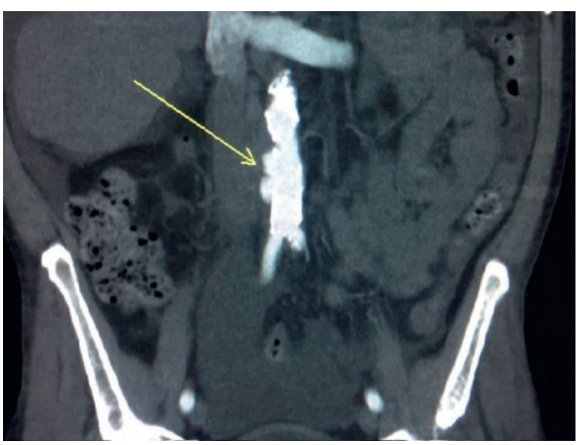

(a)

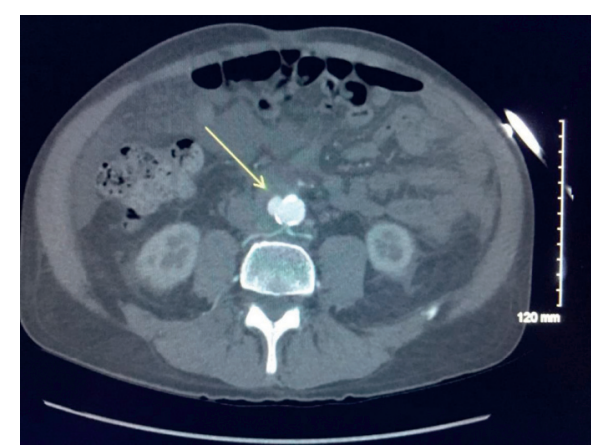

(b)

Figure 1: $(\mathrm{a}$ and $\mathrm{b}$ ) presence of multilobulated aneurysm of infrarenal abdominal aorta with aortic wall thickening and subtle fatty stranding; aneurysm is indicated by arrows, on coronal and axial images from CT of the abdomen and pelvis with iodinated contrast.

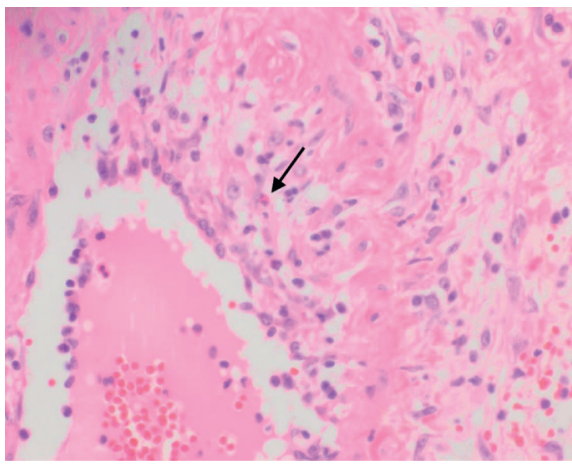

(a)

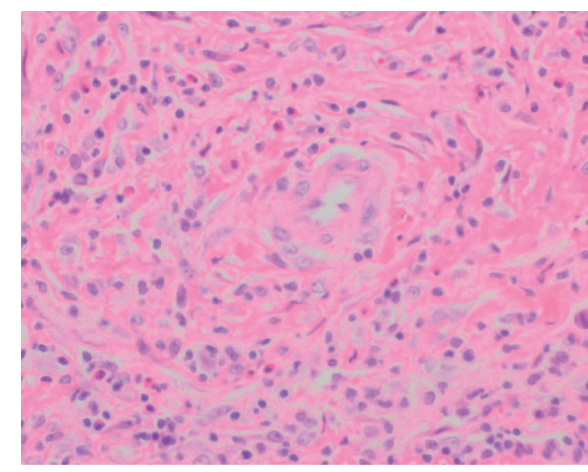

(b)

FIgURE 2: (a) Histopathologic sections of the aorta show vasculitis of small and medium vessels with occasional eosinophils within the vessel wall. (b) Infiltration of the periaortic tissue with eosinophils and other inflammatory cells.

arteritis and Takayasu arteritis but are rarely reported with Bechet's disease, sarcoidosis, Kawasaki disease, rheumatoid arthritis, ankylosing spondylitis, Systemic Lupus Erythematosus, Cogan's syndrome, IgG4 disease, granulomatous, and microscopic polyangiitis [9]. Mycotic aortic aneurysms are rare but potentially life-threatening due to the elevated risk of rupture. It can occur due to direct bacterial inoculation into the arterial wall at the time of vascular injury or from hematogenous spread of systemic infection, contiguous involvement of vessel wall from an adjacent source or due to septic emboli from the heart. Mycotic and inflammatory aneurysms both present with similar nonspecific symptoms, e.g., abdominal or back pain, weight loss, and low grade fever, and are difficult to diagnose [8]. There are few nonspecific imaging findings that can help distinguish mycotic versus inflammatory abdominal aortic aneurysm [10]. Mycotic aneurysms usually present as saccular aneurysm with irregular configuration, soft tissue stranding, and periaortic fluid or air collection. On the contrary, inflammatory aortic aneurysm presents as fusiform dilation and homogenous wall thickening with an increase in connective tissue ("Mantle sign") indicative of sclerosing inflammation [10]. CT abdomen findings in our case were more suggestive of mycotic aneurysm with its irregular configuration and soft tissue stranding. Mycotic aneurysms are treated with IV antibiotics and open surgical or endovascular repair. On the contrary, an inflammatory aortic aneurysm requires aggressive immunosuppression. Immunosuppressive therapy can lead to rapid overgrowth of organisms, potential rupture, and ultimately be fatal if used in patients misdiagnosed with mycotic aneurysms $[8,9]$. We initially did not treat this patient with corticosteroids as he did not have clinical and serological evidence of active EGPA, and his CT scan findings were more suggestive of a mycotic aneurysm. The C-reactive protein and procalcitonin levels could be high in both active vasculitis and sepsis, and one study suggested that a high procalcitonin level is more suggestive of infection rather than active vasculitis [11]. Our patient underwent an open surgical repair after the aneurysm rupture. Interestingly, the aortic biopsy findings were consistent with the diagnosis of EGPA vasculitis, and all the infectious disease workup was negative for any form of infection. Early institution of therapy would have prevented aneurysmal rupture, but we are not sure.

In summary, a diagnosis of inflammatory aortic aneurysm secondary to vasculitis should be considered in patients with a known diagnosis of EGPA, presenting with abdominal pain and having CT findings of aortic aneurysm. Early diagnosis and interventions are essential to avoid potential rupture. CT findings may not help distinguish the 
mycotic vs. inflammatory aneurysms. Our patient did not have any peripheral eosinophilia and had a negative ANCA at the time of presentation to the emergency department. This indicates that EGPA may not be active at the time of presentation, but no conclusion can be drawn based on one case report.

\section{Abbreviations}

EGPA: Eosinophilic granulomatosis polyangiitis

CSS: $\quad$ Churg Strauss syndrome

ANCA: Antineutrophil cytoplasmic antibody

AAV: $\quad$ ANCA-associated vasculitis

ESR: $\quad$ Erythrocyte sedimentation rate

CRP: $\quad$ C-reactive protein

LFT: Liver function test

GGT: Gamma glutamyl transferase

ANA: Antinuclear antibody

c-ANCA: Cytoplasmic antineutrophilic antibodies

PR3: $\quad$ Proteinase3

MPO: $\quad$ Myeloperoxidase

p-ANCA: Perinuclear antineutrophil cytoplasmic antibodies

ED: $\quad$ Emergency department

PTFE: Polytetrafluoroethylene

PAS: $\quad$ Periodic acid Schiff stain

GPA: Granulomatosis polyangiitis

MPA: Microscopic polyangiitis.

\section{Data Availability}

All the data were included in the manuscript.

\section{Conflicts of Interest}

The authors have no any conflicts of interest.

\section{References}

[1] J. C. Jennette, "Overview of the 2012 revised international chapel hill consensus conference nomenclature of vasculitides," Clinical and Experimental Nephrology, vol. 17, no. 5, pp. 603-606, 2013 Oct.

[2] T. Carels, E. Verbeken, and D. Blockmans, "p-ANCA-associated periaortitis with histological proof of Wegener's granulomatosis: case report," Clinical Rheumatology, vol. 24, no. 1, pp. 83-86, 2005.

[3] J. A. Chirinos, L. J. Tamariz, G. Lopes et al., "Large vessel involvement in ANCA-associated vasculitides: report of a case and review of the literature," Clinical Rheumatology, vol. 23, no. 2, pp. 152-159, 2004.

[4] H.-U. Schildhaus, B. Von Netzer, F. Dombrowski, and U. Pfeifer, "Atypical manifestation of a cytoplasmic antineutrophil cytoplasmic antibody (PR3-ANCA)-associated vasculitis with involvement of aortic intima and parietal endocardium," Human Pathology, vol. 33, no. 4, pp. 441-445, 2002.

[5] K. Fujii and Y. Hidaka, "Churg-Strauss syndrome complicated by chronic periaortitis: a case report and review of the literature," Internal Medicine, vol. 51, no. 1, pp. 109-112, 2012.

[6] B. Hervier, A. Masseau, C. Bossard, C. Agard, and M. Hamidou, "Vasa-vasoritis of the aorta and fatal myocarditis in fulminant Churg-Strauss syndrome," Rheumatology, vol. 47, no. 11, pp. 1728-1729, 2008.

[7] M. Prendecki and C. D. Pusey, "Recent advances in understanding of the pathogenesis of ANCA-associated vasculitis," F1000Research, vol. 7, Article ID F1000, 2018.

[8] N. Ishizaka, K. Sohmiya, M. Miyamura et al., "Infected aortic aneurysm and inflammatory aortic aneurysm-in search of an optimal differential diagnosis," Journal of Cardiology, vol. 59, no. 2, pp. 123-131, 2012.

[9] A. R. Deipolyi, C. D. Czaplicki, and R. Oklu, "Inflammatory and infectious aortic diseases," Cardiovascular Diagnosis and Therapy, vol. 8, pp. S61-S70, 2018.

[10] T. K. Moriaki Inoko and M. Inoko, "Imaging features and diagnosis of infected aortic aneurysms and inflammatory diseases involving the aorta," Angiology: Open Access, vol. 01, no. 2, p. 110, 2013.

[11] K. Herrmann, S. Schinke, E. Csernok, F. Moosig, and J. U. Holle, "Diagnostic value of procalcitonin in ANCAassociated vasculitis (AAV) to differentiate between disease activity, infection and drug hypersensitivity," The Open Rheumatology Journal, vol. 9, no. 1, pp. 71-76, 2015. 